\title{
Nuevas tareas para el profesor de español como lengua extranjera: la reflexión sobre su concepción de la enseñanza
}

\author{
Mercedes Pizarro \\ Instituto Cervantes de Bruselas
}

Recibido: 30 enero 2012 / Aceptado: 30 abril 2012

ISSN: $1697-7467$

\begin{abstract}
RESUMEN: Hace unos años los programas centrados en el alumno hicieron que una parte del debate en didáctica de lenguas extranjeras discurriera en torno al nuevo papel del profesor. Los cambios metodológicos introducidos han hecho su labor mucho más compleja, de manera que se ha impuesto una nueva discusión sobre cómo desarrollar la competencia docente para abordar las nuevas tareas. Este artículo pretende revisar los cambios producidos y aproximarse a las propuestas de la enseñanza reflexiva para enriquecer el conocimiento profesional mediante la toma de conciencia por parte del docente de su propia concepción de la enseñanza.

Palabras clave: papel del profesor, creencias del profesor, enseñanza reflexiva, competencia docente.
\end{abstract}

\section{New Tasks for the Teacher of Spanish as Foreign Language: a Reflection on Teaching Beliefs}

\begin{abstract}
Some years ago, the student-centered programs caused part of the debate related to the teaching of foreign languages to focus on the new role of the teacher. The introduced methodological changes have made the work of the teacher much more complex and resulted to a discussion on how to develop the teacher competences in order to allow tackling the new tasks. This paper aims to review some of the changes and to discuss the reflective teaching and enrich professional knowledge by making teachers aware of the learning-teaching process conception
\end{abstract}

Keywords: role of the teacher, teacher beliefs, reflective teaching, teacher competence.

\section{INTRODUCCIÓN}

En un mundo cada vez más globalizado, en una sociedad cada vez más desarrollada tecnológicamente y deseosa de acceder a la información y al conocimiento, el dominio de lenguas extranjeras se ha convertido en la llave de acceso a ese conocimiento, a la vez que el pasaporte que permite la movilidad hacia otros mundos y realidades. En este contexto, los estudios sobre las lenguas extranjeras (LE) y su aprendizaje se han convertido en áreas importantes a las que prestar atención. Ello ha quedado puesto de manifiesto no solo con la publicación a nivel europeo del Marco común europeo de referencia (en adelante MCER), 
sino también en el ámbito de las instituciones españolas y en relación con la enseñanza del español como lengua extranjera con la publicación por parte del Instituto Cervantes de los Niveles de Referencia del Español, así como a través de otra serie de proyectos de instituciones de educación superior, entre otros el proyecto ADELEEES (Adaptación de la enseñanza de lenguas al espacio europeo para la educación superior). En general, lo que revelan todas estas propuestas es un gran interés por optimizar la enseñanza y el aprendizaje de lenguas extranjeras y del español.

Todos estos proyectos, por otro lado, suponen el reflejo y la culminación de una serie de aportaciones que desde diversas áreas se ha venido produciendo y que, especialmente en las tres últimas décadas, han supuesto una importante transformación en las formas de entender la enseñanza y el aprendizaje de lenguas. La búsqueda de planteamientos más ajustados a las necesidades y realidades de las aulas ha traído cambios importantes y un gran desarrollo de la didáctica de lenguas extranjeras como disciplina, pero también ha abierto nuevos interrogantes sobre la mejor manera de abordar la enseñanza y el aprendizaje de lenguas.

Al revisar los cambios producidos se observa la influencia que las diferentes aportaciones de disciplinas como la Teoría del aprendizaje, la Psicología cognitiva, Teorías de la Adquisición de lenguas, Teoría lingüística, han hecho a lo largo de estos años y que han ido modificado y adaptado tres de los aspectos fundamentales de la didáctica de lenguas extranjeras: qué enseñar, cómo hacerlo y quién debe ser el protagonista. Pero, sin duda, lo que han puesto de manifiesto todas sus propuestas es que el proceso de enseñanza y aprendizaje de una lengua es un complejo entramado cuyos factores se encuentran relacionados. De forma que el avance y evolución en su estudio se ha debido a la idea de interdependencia de todas esas disciplinas y ello ha contribuido a configurar la actual área de estudio de la didáctica de lenguas extranjeras y, aplicado al español, a configurar una disciplina con intereses propios. Martín Peris (1998: 1) señalaba al respecto: [...] la situación actual se caracteriza por los profundos cambios que han experimentado todas las teorías y por la incorporación de la idea de interdisciplinariedad a la concepción de lo que deber ser la acción docente [...].

El presente artículo quiere llamar la atención sobre las etapas por las que ha ido transcurriendo esta evolución. Si en un primer momento el interés estuvo fundamentalmente centrado en torno a la lengua, los programas, y el alumno e, indirectamente, en el papel del profesor (Martín Peris, 2000: 1-2), actualmente la investigación en didáctica se encuentra "orientada en el profesor" y viene interesándose por explorar y analizar más en profundidad su papel como sujeto también implicado en el proceso. Hay entonces, como señala Marrero (2010: 23), una necesidad de recuperar al profesor como sujeto. Y, si bien hoy parece superada la discusión en torno al papel del profesor, planteado en el desarrollo de los enfoques comunicativos y recogida en el Marco Común Europeo de Referencia, asistimos en cambio a un nuevo debate sobre cómo desarrollar un conocimiento, habilidad y actitud docente, o mejor, la competencia docente, de manera más acorde con la realidad del profesor, que redunde en una mejor actuación y permita mayor eficacia en el proceso de enseñanza y aprendizaje de una LE.

En este sentido, un importante número de estudios se han centrado en descubrir los procesos cognitivos que están implicados en la enseñanza y el aprendizaje de la lengua. Concretamente, los centrados en la cognición del profesor señalan la importancia y la influencia que tienen dichos procesos cognitivos en la vida profesional de los docentes y, por 
lo tanto, en su quehacer. De esta forma, la exploración, la reflexión y la concienciación sobre todos los factores implicados en el proceso de enseñanza y aprendizaje de LE por parte del profesor, la práctica reflexiva de la enseñanza, puede contribuir a comprenderlo más profundamente desde la experiencia de cada docente y hacerlo más eficaz.

\section{EvoluCión Y CAMbios EN LA DidÁCTICA DE LENGUAS EXTRANJERAS}

Inicialmente, como señala M. Peris (2000: 2), el foco de interés en la didáctica de LE estuvo en el objeto: la lengua. El debate era fundamentalmente delimitar qué había que aprender como lengua extranjera. La investigación sobre el aprendizaje de lenguas en los años 70 en Europa estaba orientada fundamentalmente a especificar los contenidos de sus syllabus. Posteriormente, el concepto de competencia comunicativa y uso de la lengua condicionó los programas de enseñanza y fue hacia ellos hacia donde se desplazó el centro de la discusión y posteriormente hacia el papel del alumno como protagonista y responsable de su propio aprendizaje.

$\mathrm{Y}$, ha sido precisamente esa nueva forma de concebir la enseñanza, centrada en el alumno, la que ha supuesto un importante cambio desde el punto de vista metodológico del papel del profesor. Los nuevos programas de enseñanza de lenguas han redefinido su papel. Los enfoques comunicativos apuntan las nuevas tareas del profesor. Breen y Candlin (1980) en Richards, y Lockhart (1998: 98) describen así sus funciones:

El profesor tiene dos papeles fundamentales: el primero es facilitar el proceso de comunicación entre todos los participantes en la clase y entre estos participantes y las distintas actividades y textos. El segundo es actuar como participante independiente dentro del grupo de enseñanza-aprendizaje. Este papel se relaciona estrechamente con los objetivos del primero y surge como su consecuencia. Estos papeles implican un conjunto de papeles secundarios: en primer lugar, como organizador de recursos, siendo él mismo un recurso; en segundo lugar, como guía en los procedimientos y las actividades de la clase...Un tercer papel es el de investigador y alumno, con muchas posibilidades de contribuir con su conocimiento, habilidades y experiencia sobre la naturaleza del aprendizaje y las capacidades organizativas.

En los planteamientos metodológicos de los enfoques comunicativos es el profesor quien debe proporcionar las condiciones idóneas para que el aprendizaje pueda tener lugar de forma eficaz. [...]En él recae la tarea de diseñar y crear las condiciones y entornos de aprendizaje que permitan a los alumnos involucrarse en el propio proceso de aprendizaje [...] (Ellis, 1996).

Posteriormente, en el MCER (2002: 139-140), aunque no se aboga por una metodología concreta que determine el papel que debería desempeñar el profesor, en cambio, sí se insiste en la idea de que sean los profesores, quienes reflexionen sobre los procedimientos más adecuados a su propia situación. Se insiste en la necesidad de considerar las circunstancias específicas del entorno en el que se desarrolla su labor: 
[...] De ellos se espera que realicen un seguimiento del progreso de los alumnos y que encuentren el modo de que los alumnos reconozcan, analicen y superen sus problemas de aprendizaje, y puedan desarrollar sus capacidades individuales a la hora de aprender. Es necesario que los profesores comprendan los procesos de aprendizaje en su enorme variedad [...].

La intervención del profesor resulta por tanto decisiva e indispensable en el proceso para lograr el éxito del aprendizaje. El profesor de lengua extranjera es el responsable de conducir el proceso de aprendizaje y también favorecer el desarrollo formativo autónomo de los alumnos. Entre otras cosas, ello significa que el profesor deberá planificar clases y fijar objetivos; organizar el trabajo de los alumnos, gestionar la progresión del aprendizaje; adaptarse a las necesidades, intereses, estilos y características de los alumnos; implicar y motivar a los alumnos; seleccionar y adaptar materiales; desarrollar la competencia comunicativa; evaluar el proceso de enseñanza-aprendizaje; adaptarse a la institución, al equipo de trabajo y a la cultura del centro en el que trabaja; intercambiar experiencias con otros profesionales y participar en la comunidad profesional; colaborar con el equipo profesional en el que se integra; autoevaluar su propia actuación y reflexionar sobre su proceso formativo (Verdía, 2011: 3).

Lo que parece claro es que estas nuevas funciones suponen también para el profesor hacer frente a nuevas tareas. Tareas que suponen un nivel de complejidad importante y para las que la formación del profesor, como veremos seguidamente, es clave para abordarlas. Los planteamientos metodológicos influyen y delimitan las funciones y papeles del profesor, así pues, este aspecto debe ser tenido en cuenta en la formación de profesores. Pero, lo que muestra la investigación en didáctica es que esta debe partir no solo del conocimiento teórico, sino de la reflexión sobre sus concepciones y prácticas. De manera que una de las áreas hacia donde se orienta este nuevo modelo de profesor es hacia su faceta de investigador.

En didáctica, concretamente desde las tendencias de investigación-acción e investigación en el aula, se viene insistiendo en la necesidad de desarrollar la capacidad de investigador del profesor. Se trataría, como señala Mendoza (1998: 261), de superar el modelo de formación técnica, para llegar a la propuesta de formación profesional del docente reflexivo y crítico. La observación, la exploración y la reflexión sobre las ideas del profesor acerca del proceso y su práctica docente son una vía que puede llevar a mejorar su quehacer en el aula.

Ello pensamos que está siendo tenido muy en cuenta por parte de las instituciones dedicadas a la formación de profesores, ya que han incluido en sus planes de estudios nuevos caminos para formar a este nuevo profesor responsable de nuevas funciones y dotarle de una mayor competencia profesional a través de las nuevas herramientas para la práctica reflexiva de la enseñanza. Desarrollar estas nuevas tareas implica

[...] una forma distinta de entender el trabajo didáctico en el centro y en el aula, ya que, en este contexto, el docente deja de ser como antaño un técnico especializado en la transmisión de los conceptos de las distintas disciplinas del conocimiento y en la aplicación de métodos y estrategias diseñadas por personas ajenas a su ámbito de actuación pedagógica para pasar a ser un enseñante capacitado para diseñar su intervención educativa, reflexionar sobre la propia práctica docente y adaptarse a los cambiantes y conflictivos contextos tanto de aula como del entorno sociocultural y social [...] (C. Lomas, 1996 en Suso López, 2005: 76). 
Como se ve, si en un primer momento la didáctica de lenguas había concedido gran importancia a los aspectos relativos a cómo aprender, se puede decir que actualmente se percibe un interés creciente por ocuparse de cuestiones referidas al cómo enseñar y que afectan directamente a quienes enseñan. En ese sentido, y como quiere recoger el título del presente artículo ya no se trata de definir el papel del profesor, sino de buscar los caminos que permitan desarrollar su competencia profesional para hacer frente a los nuevos retos. Hay como veremos más adelante gran inquietud por parte de profesionales e instituciones en profundizar y avanzar en esta cuestión.

\section{El Ámbito de ele y la formación docente. Retos}

En el ámbito del español como lengua extranjera hemos asistido a una gran expansión debida a diversos factores, entre otros, el interés de países como Brasil, Grecia o Turquía por integrarlo en sus sistemas educativos, por el incremento de la comunidad hispanohablante en EE.UU. o el importante número de ciudadanos de otros países venidos a España en los últimos años en busca de un mejor empleo. Lo cierto es que estas circunstancias han hecho aumentar el interés por aprender la lengua y la enseñanza del español goza de un momento de gran vitalidad y crecimiento. Paralelamente se ha producido un importante desarrollo de su didáctica.

Ante este nuevo panorama, en el ámbito de la enseñanza de ELE, los responsables de los programas de formación de profesores buscan incluir en sus planes de estudios nuevos caminos formativos que incorporen los avances hechos durante estos años en la investigación didáctica $\mathrm{y}$, en el mundo de ELE concretamente.

En una entrevista conjunta a un importante grupo de profesionales del mundo de ELE sobre los últimos 25 años (Tolosa y Yagüe, 2009), se descubre que la constante en sus reflexiones es efectivamente la madurez adquirida por los estudios de ELE y su reconocimiento como disciplina académica y profesional, gracias a las contribuciones de profesionales e instituciones públicas y privadas. Entre los logros, todos los entrevistados destacan entre otras cuestiones, el desarrollo formativo adquirido por los profesores de esta área. Pero, también señalan la necesidad de reflexionar acerca del grado de exigencia, rigor y calidad en los planes formativos que se vienen impartiendo a los docentes de ELE y en la necesidad de explorar nuevos ámbitos en los que el profesor pueda verdaderamente articular conocimiento y experiencias.

Esteve (2009) considera que estos primeros años han sido fundamentales para el desarrollo de la formación de profesores de español y para la profesión en sí, puesto que se llevó a cabo la descripción de los conocimientos específicos de la Enseñanza del Español como Lengua Extranjera definiéndola y diferenciándola frente a otras disciplinas. Pero, quizás, como señala esta misma autora, es el momento de reconsiderar cómo se ha entendido la formación de profesores de LE. En ella parece que se ha primado la transmisión de conocimientos teóricos, sin duda necesarios y útiles para la práctica, así como la presentación de actividades para la clase, pero que no han conectado con las verdaderas necesidades del profesor y la realidad del aula. Así, en los últimos años ha surgido gran interés por encontrar nuevos ámbitos que permitan y conduzcan a esa mejora de la calidad de la enseñanza, orientándolo hacia la faceta del profesor. Y, en esta búsqueda, ha surgido una interesante discusión sobre cómo desarrollar la competencia docente del profesor de LE. 


\section{El desarrollo de la competencia docente del profesor de ELE}

En un reciente encuentro, Verdía (2011) se planteaba qué conocimientos, habilidades y actitudes debía adquirir y desarrollar un profesor de español para convertirse en un buen profesional y hasta qué punto la oferta formativa existente (másteres, cursos, etc.) responde verdaderamente a las necesidades del profesor para desarrollar su actividad profesional.

Como señalábamos anteriormente, las nuevas tareas del profesor han vuelto más compleja su labor. Entre las tareas del profesor ya no está solo la de suministrar conocimiento, sino crear condiciones favorables para que se produzca una enseñanza y un aprendizaje eficaz. Lograr esto significa por otro lado, ser capaz de poner en marcha procedimientos que le permitan planificar sus clases, gestionar el aula, investigar en el aula acerca del proceso de aprendizaje, analizar las repercusiones de su acción, evaluar el proceso y elaborar un diagnóstico sobre la situación de aprendizaje. De manera, que todo ello implica también, explorar, reflexionar y analizar de forma crítica su propia labor en aula.

Para dar respuesta a estas nuevas necesidades del docente y favorecer el desarrollo de su competencia profesional debemos considerar en qué consiste la competencia docente. Siguiendo a Verdía (2011:5), está formada por diversos factores que se encuentran estrechamente relacionados: conocimientos, habilidades y actitudes.

Por conocimientos se entienden los saberes sobre el objeto de la enseñanza, en este caso, los referidos al sistema formal del español y a la cultura y la sociedad hispana. Pero también conocimiento de las características del entorno en el que se va a producir el proceso de enseñanza y aprendizaje en los que va a tener que desenvolverse: factores educativos diversos, variables individuales, sociales y culturales y necesidades específicas. Otro aspecto relevante que deberá formar parte del conocimiento del profesor es el que tiene que ver con la didáctica del español como lengua extranjera (ELE) y como lengua segunda (L2) ${ }^{1}$ y las teorías de enseñanza y aprendizaje: adquisición de lenguas, métodos y enfoques de la enseñanza, desarrollo curricular, gramática pedagógica, ciertos conocimientos de psicología y pedagogía.

Por otra parte, todo profesor debe desarrollar habilidades para desenvolverse en el aula, en el centro de trabajo y en la comunidad profesional. Estas capacidades tienen que ver con la práctica profesional y están más directamente relacionadas con las tareas que realiza el docente dentro y fuera del aula: habilidades docentes (técnicas de gestión del aula, de planificación, de evaluación...), destrezas de comunicación (desarrollo de habilidades para transmitir conocimientos, mejorar la expresión, habilidades interculturales, destrezas profesionales, habilidades interculturales (desarrollo de habilidades para servir de intermediario y de mediador intercultural, dado que va a trabajar con alumnos de diferentes lenguas y culturas. Así como adquirir destrezas profesionales (capacidad para trabajar en equipo, gestionar recursos compartidos,....)

\footnotetext{
${ }^{1}$ Aunque en ocasiones se emplean los dos términos para aludir a un mismo fenómeno, sin embargo en el área de adquisición de lenguas muchos estudiosos prefieren hacer la distinción entre LE y L2. Según Santos Gargallo (1999: 21), L2 es aquella que cumple una función social e institucional en la comunidad lingüística en que se aprende. En cambio, LE es aquella que se aprende en un contexto en el que carece de función institucional y social en la comunidad en que se aprende.
} 
Además, los profesores al igual que otros profesionales poseen actitudes determinadas frente a lo que hacen, sobre el proceso de enseñanza y aprendizaje, sobre su profesión, los alumnos, los demás profesionales, la institución para la que trabajan y él mismo.

Ahora bien, no se trata de adquirir conocimientos, desarrollar habilidades y promover actitudes. Ello no basta para ser competente:

[...] un profesional es competente no sólo porque manifieste conductas que expresan la existencia de conocimientos y habilidades que le permiten resolver adecuadamente los problemas profesionales, sino también porque siente y reflexiona acerca de la necesidad y el compromiso de actuar en correspondencia con sus conocimientos, habilidades, motivos y valores, con flexibilidad, dedicación y perseverancia, en la solución de los problemas que de él demanda la práctica profesional. [...] (Esteve, 2009: 6).

Los docentes poseen una visión personal de la enseñanza. Muchos profesores crean sus propios papeles en el aula basándose en sus propias teorías de enseñanza aprendizaje y el tipo de interacción en el aula que mejor se adecúa a sus teorías (Richard y Lockhart, 1998: 99). Generan sus propias concepciones acerca de la enseñanza y adoptan determinadas actitudes con respecto al proceso de enseñanza y aprendizaje de la LE.

La actitud de un profesor de segundas lenguas hacia el propio proceso de enseñanza aprendizaje es una combinación entre lo que sabe y cree sobre del proceso de enseñanza y sobre cómo aprenden los alumnos, lo que siente ante determinadas situaciones educativas y su predisposición a actuar de una determinada manera. En la actitud ante la enseñanza están implicadas tres dimensiones que operan de manera interrelacionada: afectiva (sentimientos evaluativos y preferencias), cognitiva (opiniones y creencias ${ }^{2}$ ) y conductual (acciones manifiestas y declaración de intento) (Clark y Peterson, 1986; Lynch, 1989 en Richard y Lockhart, 1998: 34).

Así, aunque muchos profesores pueden haber sido formados en una determinada línea metodológica o se les hayan enseñado determinados contenidos y habilidades pedagógicas, la forma en la que enseñan es a menudo una interpretación personal de lo que piensan y sienten: Las creencias profundamente enraizadas que tienen los profesores sobre la forma en que se aprende una lengua impregnarán sus actuaciones en el aula más que el método concreto que estén obligados a adoptar o el libro de texto que sigan (Willians y Burden, 1999: 65).

Según esto, si de lo que se trata es verdaderamente de mejorar y hacer más eficaz la enseñanza de ELE, ello debe también pasar por un cambio en la actitud de los profesores. $\mathrm{Y}$, consecuentemente, como señalan algunos estudiosos del tema, se impone un cambio que contenga también instrumentos para poder intervenir desde la formación en relación con las actitudes del profesor.

\footnotetext{
${ }^{2}$ La investigación sobre cognición viene utilizando el término creencias para referirse a las ideas que docentes y aprendices poseen sobre cuestiones relacionadas con la enseñanza y aprendizaje. Aquí se emplea el término indistintamente junto con los términos pensamiento, percepciones, concepciones, teorías implícitas, representaciones, etc., no obstante, en algunos autores, estos aluden a conceptos diferentes.
} 
[...] parece que todo el debate en el ámbito de la formación debe girar en torno a un concepto clave: el de «cambio» o «transformación». Las investigaciones más recientes en el campo de la formación de profesorado (Korthagen, 2001; Libermann, 1998; Wallace, 1991) apuntan una idea importante al respecto: si se desea fomentar un proceso de formación que realmente desemboque en un cambio cualitativo, es importante y necesario adoptar un modelo más ligado a la realidad del profesor en formación, que parta de sus experiencias vividas y de la realidad de su futuro profesional; un modelo, en definitiva, que parta de la persona misma y no del saber teórico[...] Esteve (2004: 9).

$\mathrm{Al}$ comienzo de esta comunicación recordábamos el cambio de papeles y funciones que la evolución didáctica había supuesto para el profesor de lenguas extranjeras. Ahora bien, esos cambios implicaban también una nueva forma de concebir la enseñanza, y ello por su parte requiere una nueva actitud para llevarlos adelante. La nueva perspectiva adoptada por los enfoques comunicativos, intentan promover una actitud docente determinada, requieren de profesores flexibles, dinámicos, activos, pedagógicamente formados, sin miedo a la innovación y a la investigación sobre su propia acción, que utilicen diferentes métodos y adapten su estilo de enseñanza al momento y contexto particular.

Martín Sánchez, (2007: 28) señala por su parte que el perfil de profesor que promueven estos enfoques debe poseer flexibilidad, adaptación, sensibilidad, capacidad para dar respuestas a los problemas que se encuentra en el aula (profesionales y emocionales), empático, que sea capaz de observar lo que está ocurriendo en el aula, no dominante, ni directivo, ni autoritario, que no intente influir directamente en la vida de su alumnos, que intervenga para que el alumno participe y aprenda, con una actitud positiva, abierto al cambio y a la innovación, implicado en su trabajo, que posea una fuerte y profunda motivación.

Adoptar esa nueva actitud, esa nueva forma de ser y estar en clase requiere también de intervenciones y, como se viene insistiendo parece claro que no se puede promover una actitud determinada desde un planteamiento de formación con contenidos teóricos, sino que lo que se requiere son otros instrumentos de intervención para lograr una formación docente eficaz. Y, aunque creencias y actitudes son reacias a cambiar, sin embargo, a pesar de esa dificultad, existen estudios sobre la noción de cambio en las creencias de los profesores que ponen de manifiesto cómo el cambio de las creencias precede a los cambios en la práctica (Richards, 2001). En ese sentido, el análisis y la reflexión sobre las propias concepciones del profesor y la observación de su práctica pueden contribuir a construir de forma integral su competencia docente, proporcionándole otro tipo de conocimiento, un conocimiento desde el docente, que le permita verdaderamente incrementar su conocimiento de manera más personal del proceso de enseñanza aprendizaje de la lengua y de esa forma contribuir a construir su competencia de profesor.

La construcción de la competencia del profesor debe por tanto tener en cuenta también todos los aspectos referidos a la cognición del profesor: pensamientos y creencias, representaciones mentales de la realidad, valores, saberes personales, unos saberes compartidos, esquemas de percepción, ya que todos ellos están presentes en su toma de decisiones e influyen en su práctica.

En la reflexión sobre el oficio de enseñar, la figura del practicante reflexivo, propuesta por Schön, se impone cada vez con más fuerza. Los saberes racionales no bastan para hacer frente a la complejidad y a la diversidad de situaciones laborales. Por 
este motivo la principal apuesta consiste en recuperar la razón práctica, es decir los saberes de la experiencia basada en un diálogo con lo real y la reflexión en la acción y sobre la acción. La práctica reflexiva tiene como objetivo coparticipar y hacer dialogar entre sí estos diversos saberes (Perrenoud, 2004).

Como señala Marrero (2010: 31) citando a Gimeno (1998) la reflexión es la clave, el motor, del conocimiento del oficio. Gracias a la reflexión, continúa el mismo autor, citando a Schön (1983 y 1987) el conocimiento de oficio supone experiencia transformada, de la misma manera que transformar la experiencia supone reflexionar sobre la misma.

\section{EL DOCENTE Y LA ENSEÑANZA REFLEXIVA}

El concepto de práctica reflexiva de la enseñanza parte de una visión constructivista del aprendizaje cuyos planteamientos metodológicos relacionan praxis y teoría. Aplicado al ámbito de la didáctica este enfoque se basa en el análisis de actitudes, creencias y prácticas del docente a través de la reflexión crítica de sus concepciones y de su práctica con el fin de mejorarla.

[...]Uno de los giros más recientes en la enseñanza de lenguas extranjeras es el desplazamiento desde "métodos" y otras visiones "externas" o "de arriba a abajo" de la enseñanza a un enfoque que busque comprenderla en sus propios términos. Dicho enfoque comienza por los profesores mismos y los procesos reales de enseñanza, y trata de obtener una mejor comprensión de estos procesos explorando, con los profesores, lo que hacen y por qué lo hacen [...]. (Lockhart y Richards, 1998: 8).

La práctica reflexiva se basa en la presuposición de que un profesor debe estar informado y poseer un conocimiento extenso de la enseñanza. Para lograr esto, la introspección puede ser de gran ayuda, ya que muchas veces los profesores desconocen en gran medida lo que ocurre en el proceso de enseñanza. A menudo no son conscientes del tipo de enseñanza que practican y no basta con la experiencia. Muchos profesores generan estrategias y rutinas sin que medie reflexión (Parker, 1984 en Lockhart y Richards, 1998: 4). La reflexión crítica puede promover una comprensión más profunda de la docencia (Barlett, 1990; Wallace, 1991 en Lockhart y Richards, 1998: 14).

Se trata de una visión según la cual el conocimiento sobre la práctica docente debe ser un conocimiento que surge del docente, y no de un conocimiento ya creado con anterioridad por terceros y transmitido por ellos. (Esteve, 2009: 5).

Quizás el constructivismo, como señala Glasersfeld, 1995 (en Williams y Burden, 1999: 60), no puede decir al profesor qué cosas nuevas tiene que hacer, pero puede sugerir por qué son contraproducentes ciertas actitudes y procedimientos. La enseñanza, al igual que el aprendizaje, debe suponer que los profesores comprendan las situaciones en las que se encuentran. 
Se aboga entonces, señalan González y Atienza (2010: 40) por un docente reflexivo que desde la docencia indague en las teorías existentes para acercarlas a la problemática observada. Además, señalan estas dos autoras, el proceso es inverso al tradicionalmente considerado: es la docencia la que busca en la teoría, y no la teoría la que busca incidir sobre la docencia, entendiendo de este modo que teoría y práctica se retroalimentan.

Los investigadores han utilizado varios métodos diferentes para intentar entender el sentido que los profesores dan a su trabajo: el estudio del pensamiento y estudios etnográficos de sus actuaciones. Para ello, el profesor puede utilizar diversos instrumentos para recoger todo lo que ocurre en el aula, para su posterior análisis e interpretación: el portfolio del profesor, los diarios de clase, las entrevistas, las encuestas, las grabaciones de aula, etc., (Richards y Lockhart, 1998). Todos estos instrumentos, que actualmente forman parte de la investigación didáctica, recogen y plasman las formas que tienen los profesores de pensar la enseñanza de ELE y las formas de actuar en el aula. Todo ello nos hará tomar conciencia de lo que hacemos y por qué lo hacemos y ello sin duda incrementará nuestro conocimiento permitiéndonos crecer y mejorar como profesores.

\subsection{El estudio del pensamiento del profesor de LE}

Según lo señalado hasta aquí, del análisis de las creencias y pensamientos que subyacen a las acciones de los profesores podremos llegar a comprender mejor su concepción de la enseñanza: [...] en lo que se refiere al pensamiento de los profesores acerca de la enseñanza y el aprendizaje, lo que se busca no es tanto perfilar la figura del profesor eficaz, sino explicar y comprender los procesos de enseñanza [...] (Halkes y Olson, 1984 en Lockhart y Richards, 1998: 34).

Como señalan los estudiosos del tema, en las últimas dos décadas, la investigación didáctica se ha centrado en el estudio de los procesos cognitivos y su influencia en la vida profesional de los docentes. Ello ha generado una importante línea de investigación. Borg (2003) recoge las cuestiones fundamentales hacia donde se dirigen las investigaciones sobre la cognición de los profesores. Entre ellas señala: ¿En torno a qué giran los pensamientos del profesor y cómo se desarrollan? ¿Cómo influyen en su concepción de la enseñanza y aprendizaje? ¿Cómo influyen en la práctica de clase?

Para estos nuevos planteamientos didácticos, las creencias de los profesores ocupan un lugar importante ya que son un aspecto cuyo estudio y análisis puede proporcionar información interesante que redunde en una mejora de las actuaciones en el aula. Pero, ¿qué entendemos por estudio de la cognición, de las creencias? Para Borg (2003), cognition es el marco en el que las creencias están incluidas junto a conceptos como conocimientos, representaciones, imágenes, actitudes, concepciones, percepciones, teorías... y que configuraría lo que el profesor conoce sobre el proceso de enseñanza y aprendizaje. Por su parte, Ramos (2005:18) propone la siguiente definición de creencias:

[...]Las creencias son ideas relativamente estables que tiene un individuo sobre un tema determinado, forjadas a través de su experiencia personal bajo la influencia de un proceso de construcción social, agrupadas en redes o sistemas, de cuya veracidad está convencido y que actúan como un filtro a través del cual percibe e interpreta el mundo que lo rodea, tomando sus decisiones de acuerdo con ello [...]. 
Cambrá Ginés (2009: 20) habla de vida mental de los profesores y señala:

Les travaux développés depuis les années soixante-dix sur la vie mentale des professeurs (Calderhead, 1996 ; Clandinin \& Connelly, 1987 ; Clark \& Peterson, 1986 ; Elbaz, 1985 ; Shavelson \& Stern, 1981) ont établi que les représentations permettent aux enseignants d'attribuer un sens à l'expérience, de guider les comportements et de gérer les dilemmes. Elles sont contextualisées, forgées à partir du vécu, chargées d'affectivité, anecdotiques et même contradictoires liées aux valeurs et ne coïncident pas toujours avec les savoirs scientifiques; elles ont un caractère personnel et spécifique mais aussi socioculturel et sont diffusées par les institutions et les médias.

Lo que parece claro es que los profesores están muy influidos por sus creencias (Williams y Burden, 1997:65) y que estas están relacionadas con sus valores, su visión del mundo, y con el concepto que tienen de su posición en el mismo (Argyris y Schön, 1974). Los estudios existentes señalan incluso que estas influyen más que los conocimientos que tienen los profesores sobre cómo planifican sus clases, en el tipo de decisiones que toman y en su labor general en el aula (Pajares, 1992) y que son reacias a cambiar.

La complejidad del estudio de las creencias, no obstante, también ha sido puesta de manifiesto ya que se trata de un aspecto difícilmente observable en el aula. Algunos autores utilizan la metáfora del iceberg al hablar de este tema. Los trabajos de investigación sobre la procedencia de las creencias de los profesores incluyen entre otras razones, las experiencias previas, tanto como estudiantes de lenguas, como la propia experiencia sobre qué planteamiento didáctico o enfoque metodológico funciona.

En relación con los tipos de creencias del profesor, Richards (2001) especifica y concreta las cuestiones hacia donde se dirigen las creencias respecto del aprendizaje y la enseñanza de una LE: creencias sobre el papel del profesor, sobre los alumnos, sobre la lengua que enseñan, sobre el programa y el currículo, sobre la enseñanza de lenguas como profesión, sobre el aprendizaje y la enseñanza de la lengua. Dentro de estas incluye: creencias respecto de la gramática y su enseñanza, del léxico, de la enseñanza de las destrezas, de la corrección de errores y de la práctica de clase.

Las creencias de los profesores en torno al proceso de enseñanza aprendizaje de una LE se relacionan directamente con lo que los docentes consideran que es eficaz para favorecer el aprendizaje e influye directamente en su forma de enseñar. Brindley (1984) señala que generalmente las concepciones de los profesores con respecto al aprendizaje suelen formarse a partir de su experiencia docente o también como señala Freeman (1992), a partir de su experiencia como alumnos de lenguas. Así, como consecuencia de esa experiencia pasada podemos encontrarnos con profesores que optan por una forma de aprender en la que consideran fundamental el conocimiento explícito de la gramática, frente a otros que prefieren facilitar oportunidades para usar lengua; profesores que optan por un estilo o estrategia para sugerir a los alumnos, tal como deducir significados por el contexto, frente a memorizar vocabulario; trabajar en pequeños grupos, frente a otros que consideren fundamental el trabajo individual; profesores que opten por un papel específico para desempeñar en el aula como, por ejemplo, el papel de profesor como transmisor de conocimientos frente al papel de facilitador del aprendizaje, entre otros. 
Breen, 1991 (en Borg, 2003) en una investigación realizada (The implicit theories of experienced language teachers) señala nueve principios que los profesores consideran facilitan el aprendizaje de la lengua: atención a la forma de la lengua (Focus on form), atención al vocabulario o a los significados; permitir a los estudiantes usar la lengua, dirigir las capacidades de procesamiento mental de los estudiantes, tener en cuenta el entorno afectivo del estudiante, dirigir las necesidades e intereses de los estudiantes, controlar el progreso de los estudiantes y facilitarles retroalimentación, promover la responsabilidad y la autonomía de los estudiantes, llevar adelante las lecciones y el grupo.

Por otro lado, un estudio llevado a cabo entre profesores de ELE para indagar en sus creencias acerca de la gramática y su enseñanza (Pizarro, 2012) ponía de manifiesto lo que venimos abordando. Los profesores, aunque pueden haber sido formados según unos determinados principios metodológicos, su forma de estar en el aula es generalmente una interpretación personal que refleja su forma de concebir la enseñanza. Su labor en el aula resulta una mezcolanza de creencias y actuaciones, muchas veces contradictorias, que va más allá de su formación teórica. Los datos obtenidos a través de un cuestionario mostraron que posee opiniones propias sobre lo que puede funcionar en el aula, por lo que incorpora todo tipo de recursos de que dispone, combinando para ello actividades de diversa naturaleza, en la búsqueda de eficacia en el aprendizaje. Más allá de las pautas de un determinado enfoque metodológico, la creencia sobre lo que consideran que es eficaz prevalece, de ahí la mezcolanza de actividades y planteamientos hacia la gramática y lo ecléctico de sus ideas.

\section{Conclusiones}

Hemos visto a lo largo de la evolución producida en los últimos años en el ámbito de la didáctica que en la enseñanza y el aprendizaje de una lengua no basta con priorizar un factor u otro, sino que este proceso es un complejo entramado en el que intervienen muchos elementos a los que vienen a sumarse las ideas, concepciones personales y actitudes de los protagonistas del mismo. En los últimos años, la investigación en didáctica ha revelado que todos los aspectos relacionados con la cognición del profesor influyen y condicionan su práctica en el aula. De manera que para mejorar la práctica del aula se requiere también de instrumentos y mecanismos que permitan actuar en las ideas y concepciones del profesor.

Como se señalaba al comienzo de este artículo, el avance de la didáctica hizo que cambiaran también los principios metodológicos desde los cuales los profesores habían venido actuando. Los enfoques comunicativos trajeron una nueva forma de afrontar la enseñanza. En ese contexto, inicialmente la didáctica de LE estuvo centrada en delimitar los contenidos de la enseñanza de LE, enfocando la enseñanza hacia el alumno y definiendo los papeles que alumno y profesor debían asumir en los nuevos planteamientos metodológicos. Tras todos esos cambios, lo que surge es la necesidad de dotar a los profesores de una mejor formación que le permita afrontar las nuevas tareas. En ese sentido creemos que es importante prestar atención, como actualmente se viene haciendo, a las cuestiones que tienen que ver con el profesor y su desarrollo profesional, ya que el docente es también una pieza clave del proceso.

Por otro lado, los planteamientos teóricos desde los que viene desarrollándose la formación docente no parecen ser suficientes para abordar ese componente cognitivo del que 
hablamos. Es importante desarrollar nuevas vías para lograr una intervención desde la formación docente que redunde verdaderamente en una mejora de la calidad de la enseñanza. La práctica reflexiva aplicada a la enseñanza, entendida como un conjunto de herramientas al servicio del análisis y la reflexión, debe ser tenida en cuenta. A través de los diversos instrumentos con los que opera (diarios de clase, portafolio, cuestionarios, grabaciones...) el profesor logra establecer puentes entre su conocimiento sobre la enseñanza y su realidad en el aula. Incorporar la reflexión a la práctica docente llevará al propio profesor a ser partícipe de la construcción de su propia competencia profesional. La reflexión sobre su pensamiento y su práctica docente le permitirá tomar conciencia sobre ello, de manera que pueda mejorarla.

\section{Bibliografía}

Alsina, Á. y Esteve O. (2009). “Cómo aprender competencias profesionales durante la formación inicial de maestros? Algunas aportaciones desde el aprendizaje realista”. Disponible en: http://dugi-doc.udg.edu/bitstream/10256/1952/1/136.pdf >

Borg, M. (2001). "Teacher's beliefs". ELT Journal Volume 55/2. Oxford University Press.

Borg S. (2003). "Teacher cognition in language teaching: A review of research on what language teachers think, know, believe, and do". Cambridge U. Press. Disponible en: http://www. personal.leeds.ac.uk/ edusbo/cognition/Articles/Language\%20Teaching\%2036- 2.pdf.

Cambrá Giné, M. (2009). "L'accueil des Enfants Étranger et l'Éducation Linguistique en Catalogne : Représentations d'une Enseignante d'École Rurale", en Santé et Éducation de l'enfance, 1 (1): 19-31. Simon Fraser University, Colombie-Britannique, Canada.

Consejo de Europa (2002). Marco Común Europeo de Referencia para las Lenguas: Aprendizaje, Enseñanza y Evaluación.

Esteve, O. (2004). "Nuevas perspectivas en la formación de profesorado de lenguas: hacia el «aprendizaje reflexivo» o «aprender a través de la práctica»". Disponible en http://cvc. cervantes.es/ensenanza/biblioteca_ele/publicaciones_centros/PDF/bremen_2004/02_esteve. pdf.

Esteve, O., Melief, K. y Alsina, A. (2010). "Creando mi profesión. Una propuesta para el desarrollo profesional del profesorado". Cap. I. Barcelona: Ediciones Octaedro. Disponible en: http://www.octaedro.com/pdf/10117.pdf.

Farrell, T.S.C. (2005). "Conceptions of Grammar Teaching: A case study of Teachers' Beliefs and Classroom Practices". Teaching English as a second or foreign language. Disponible en: http://tesl-ej.org/ej34/a9.pdf.

González, V. y Atienza, E. (2010). "El docente reflexivo: ventajas e inconvenientes del portafolio docente". Revista Lenguaje, 35-65.

Marcelo García, C. (1992). "Cómo conocen los profesores la materia que enseñan. Algunas contribuciones de la investigación sobre el conocimiento didáctico del contenido". Actas del Congreso "Las didácticas específicas en la formación del profesorado". Santiago de Compostela. Disponible en: http://www.inet.edu.ar/programas/formacion_docente/biblioteca/ politica_educativa/MARCELOGARCIAComo_conocen_docentes.pdf.

Marrero, J.E. (2010). "Escenarios, saberes y teorías implícitas del profesorado", en Marrero, J.E. (coord.): El pensamiento reencontrado. Barcelona: Octaedro Universidad, 9-47.

Martín Peris, E. (2009). "Los modelos pedagógicos y la práxis en el aula en el Simposio «25 años de ELE»". Madrid: Instituto Cervantes. 
Martín Peri, E. (2000). "La enseñanza centrada en el alumno: Algo más que una propuesta políticamente correcta". Disponible en http://www.upf.edu/pdi/dtf/ernesto.martin/archivos/ articulos/ensenanza_alumno.pdf.

Martín Peris, E. (1998). "El profesor de lenguas extranjeras: papel y funciones". Disponible en: http://www.upf.edu/pdi/dtf/ernesto.martin/archivos/articulos/ensenanza alumno.pdf.

Martín Sánchez, M. Á. (2007). "El profesor de E/LE: personalidad, motivación y eficacia", en Ogigia. Revista electrónica de estudios hispánicos, $\mathrm{n}^{\circ}$ 1: 17-30. Disponible en: http://www. ogigia.es/OGIGIA1_files/MARTIN.pdf.

Mendoza, A. (1998). "Marco para una Didáctica de la Lengua y la Literatura en la formación de profesores", en Rev. Didáctica, 10: 233-269. Madrid. Servicio de publicaciones U. Complutense. Disponible en: http://www.ucm.es/BUCM/revistas/edu/11300531/articulos/ DIDA9898110233A.PDF.

Perrenoud, Ph. (2004). Desarrollar la práctica reflexiva en el oficio de enseñar. Barcelona: Gráo. Resumen disponible en: http://www.unige.ch/fapse/life/livres/alpha/P/Perrenoud_2004_B. html.

Pizarro, M. (2012). "Un acercamiento a las creencias de los profesores de LE acerca de la gramática y su enseñanza", en Revista Mosaico, no 13. Junio, 2012. Bruselas. (En prensa).

Pizarro, M. (2010). "Un acercamiento a las creencias de los profesores de LE" en Revista Decires, vol. 12, $\mathrm{n}^{\circ}$ 15, segundo semestre, 2010, pp. 27-38. México. Disponible en http:// revistadecires.cepe.unam.mx/articulos/art15-3.pdf.

Pujolá, J. y González, M.V. (2007). "Portafolio reflexivo del profesor". Disponible en http:// prpele.wordpress.com.

Ramos Méndez, C. (2005). Ideaciones de estudiantes universitarios alemanes sobre su proceso de aprendizaje de español como lengua extranjera ante una enseñanza mediante tareas. Tesis doctoral de la Universidad de Barcelona.

Richards, J. (2001). "Exploring teacher's beliefs and the processes of change". Disponible en: http://www.professorjackrichards.com/pdfs/exploring-teacher-change.pdf.

Richards, J. (2003). "30 Years of TEFL/TESL: A personal reflection". Disponible en: http://www. professorjackrichards.com/pdfs/30-years-of-TEFL.pdf.

Richards, J. y Lockhart, C. (1998). Estrategias de reflexión sobre la enseñanza de idiomas. Cambridge: Cambridge University Press.

Santos Gargallo, I. (1999). Lingüistica aplicada a la enseñanza/aprendizaje del español como lengua extranjera. Madrid: Arco/Libros.

Suso López, J. (2005). "El currículum español actual de lengua extranjera: Valoración, crítica y propuestas". Revista Interuniversitaria de Formación del profesorado, año/vol. 19, núm. 002, Zaragoza: Universidad de Zaragoza, 75-110.

Verdía, E. (2011). "De la adquisición del conocimiento al desarrollo de la competencia docente: profesionalización de los profesores de ELE”, en Actas II Encuentros Comillas. Disponible en http://www.scribd.com/doc/48200528/II-Encuentros-Comillas-Nov-2010-vDEFScribd.

Williams, M. y Burden, R.L. (1997). Psicología para profesores de idiomas, Madrid/ Cambridge: Cambridge University Press.

Yagüe, A., et al. (2009). "Entrevista plural: 25 años de ELE”. Disponible en http://marcoele.com/ descargas/9/veinticinco.pdf. 